\title{
Engineering Analysis of Design of Mammalian and Avian Femurs
}

Joan C. Sharp

Department of Biological Sciences, Simon Fraser University, Burnaby, BC, Canada, V5A 1S6 (jsharp@sfu.ca)

\section{ABSTRACT}

Natural selection can change structures over time by differential survival and reproduction of individuals with heritable phenotypic variation, increasing adaptation of organisms to their environment.

We can seek insight into organismal structures by applying engineering principles of good design, by (1) identifying the purpose of the structure, (2) determining the physical

constraints acting on it, and (3) calculating costs and benefits if the structure was changed. Mammalian and avian femurs are hollow tubes, not solid rods. A tube is an excellent shape for a structure that must resist bending and breaking, yet be as light as possible. A tube is stronger than a solid rod of the same mass. A tubular bone of extennal diameter dhas an internal cavity with internal diameter $k d$. The factor $k$ is 0 if a bone is solid or close to 1 if a bone is very thin-walled. Note that avian or mammalian bones do not actually range across these $k$-values. The mass per unit length of a series of bones of identical strength but differing values of $k$ can be calculated. Students determine whether $k$ values of various mass for femurs of a given strength.

\section{ADAPTIVE DESIGN OF ORGANISMS}

Natural selection may act to change structures over time by the differential suivival and reproduction of individud with heritable variants of the structure. The result is increasing adaptation of organisms to their environment. In a lab on adaptive design, we examine a number of constraints on natural selection, including tradeoffs between costs and benefits of a structure and physical constraints imposed by the laws of physics. Natural selection can only build well-designed structures by rical constraint is also fundamental part of evolution.

We can seek insight into the structures that we see in the biological world by applying engineering principles of good design. To apply these principles, we must (1) identify the purpose of the structure, (2) determine the physical constraints acting on it, and (3) calculate the costs and benefins if the stucture was to be changed. In this lab, students carry out an engineering analysis of the design of mammalian and avian femurs.

\section{ENGINEERING ANALYSIS OF DESIGN OF MAMMALIAN FEMURS}

The long bones in the legs of mammals and birds are hollow tubes, not solid rods. What is the advantage of this design? Bones should be strong. Obviously, a broken leg poses a tremendous hazard to an animal. However, bones should also be light. Heavy bones may be strong, but they require large energy expenditure for lifting an movement. A tube is an excellent shape for a structure that must resist bending and breaking and yet must be as light as possible. A tube is stronger than a solid rod of the same mass.

Figure 1 shows a section through a tubular bone of external diameter $d$. It has an internal cavity with internal diameter $k d$. The factor $k$ (the ratio of the internal diameter of the tube to its external diameter) can have any value from 0 , if the bone is solid, to close to 1 , if the bone has a very thin wall.

The mass per unit length of a series of bones of identical strength but differing values of $k$ can be calculated and are graphed in Figure 2 Streng is defined as the ability to withstand equal bending without breaking. Note that these values were calculated, not measured. There are no bones (bird or mammal) that range across these $k$ values.

Does $\mathrm{k}$ have an optimum value? In the graph, the line marked "bone only" shows that the thinner ), the lighter the bone will be.

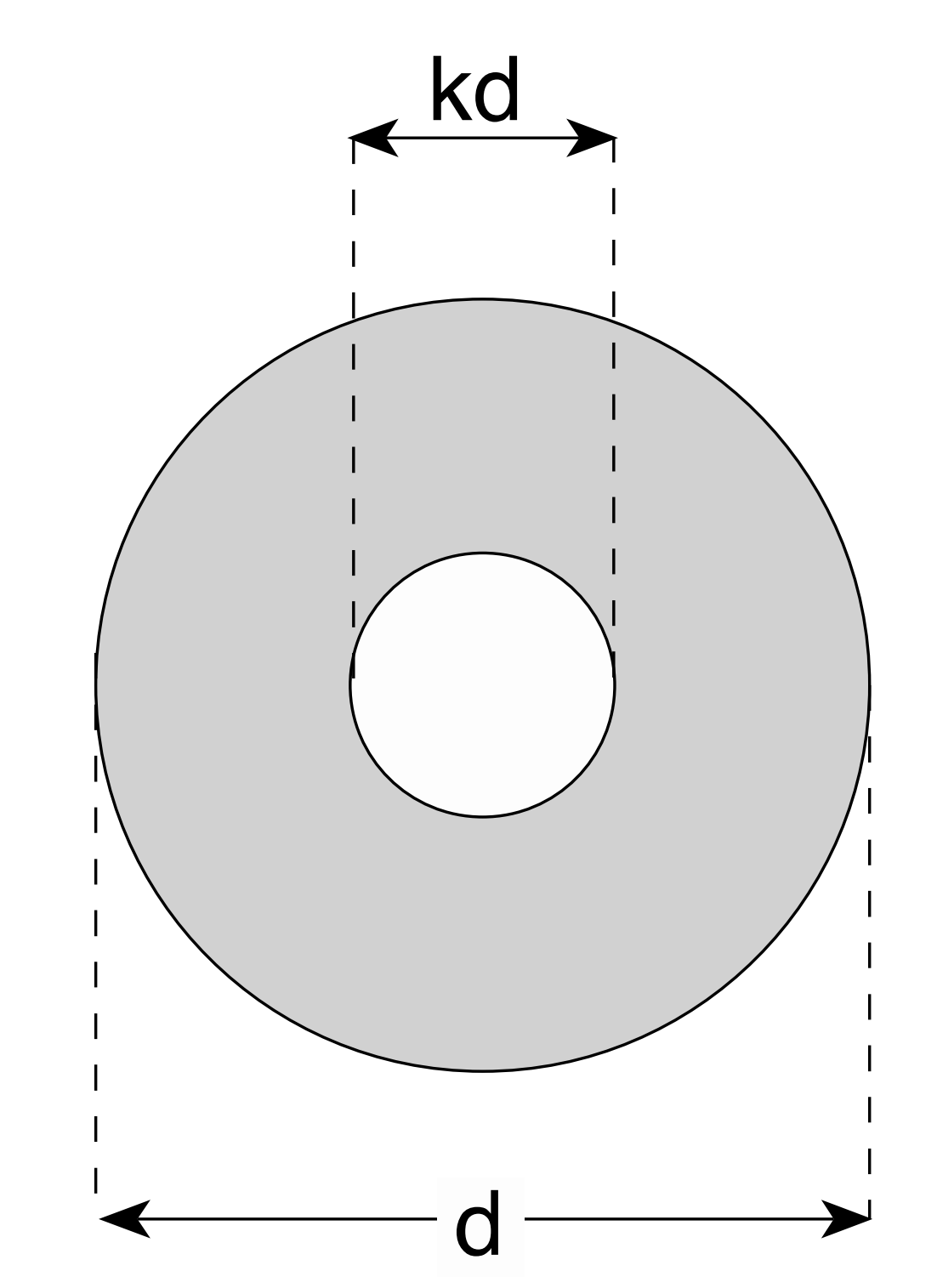

Figure 1. Tubular bone design.
In mammals, the long bones are filled with marrow, approaches 1 , the mass of marrow increases rapidly, as indicated by the line marked "marrow." The masses of the bone and marrow can be added to give the total mass per unit lengt of the bone. This line is drawn on the graph as the line "bone and marrow." As shown in Figure 2, his value is at a minimum when $k=0.63$.

Thus, a marrow-filled bone of a given strength with $k=0.63$ should have a minimum mass per unit length. This optimum holds true for any mammalian bone. Although the femurs of a shrew and an elephant would have very different diameters $(d)$ and strengths, the shape of the graph for either bone would be the same. In either animal, the mass per unit strength of the femur would be minimized at a $k$-value of 0.63 . Values of $k$ are quite close to the optimum value for a variety of mammals.

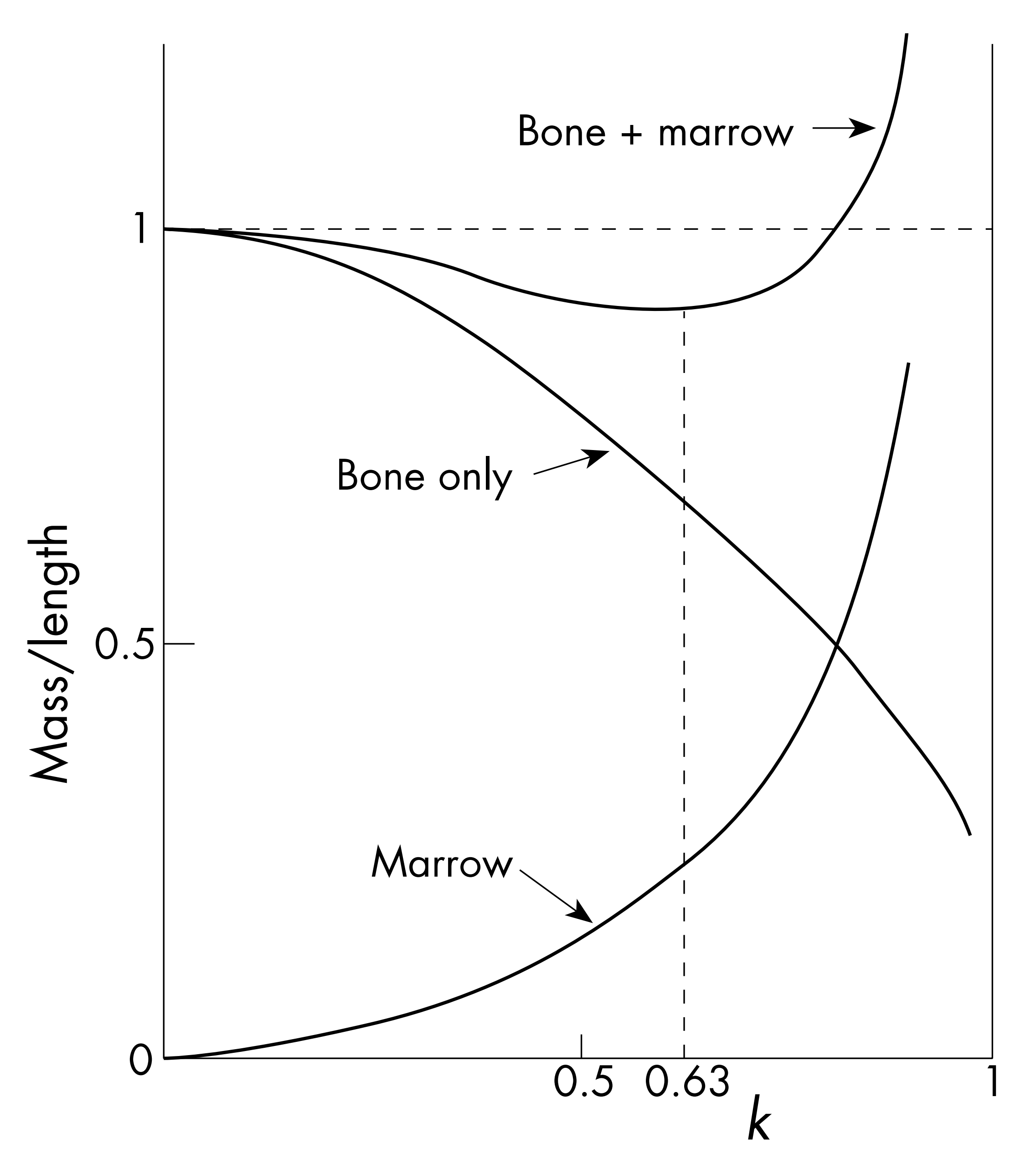

Figure 2. Graph of mass/length against the parameter $k$ for bones that can withstand equal taken as 1 for solid bones $(k=0)$

\section{PHYSICAL CONSTRAINTS ON ADAPTIVE DESIGN}

Natural selection operates within a number of constraints imposed by the laws of physics.

The force of gravity acts on all organisms. Acceleration due to gravity is a constant, but the consequences of a fall from a height depend on the mass of an organism. A mouse can easily survive a fall from a height that would kill a man, while a spore released at the same height may actually remain airborne. The surface tension of water is very high relative to other liquids, due to water's great cohesion. This gives an advantage of immense importance to vascular plants, which rely on cohesion to carry water molecules to their topmost leaves. Water striders take advantage of water's surface tension to support their weight.

The design of organisms reflects the properties of the physical world in which life has evolved. The basic laws of physics impose powerful constraints on the range of designs possible for living organisms. The also offer opportunities to living organisms.

\section{BIRD FEMURS}

Most bird bones are filled with air and buttressed with internal struts to prevent buckling. What is the optimal $k$-value for a bird femur? The analysis of the mass/length of a bird femur is approximated by considering the "bone only" line on Figure 2. As $k$ of a bird femur increases, the mass/length of the bone decreases. Thus, optimal $k$ values for birds would be expected to be higher than optimal $k$-values for mammals.

tt a very high $k$-value, a very thin walled bone may be vulnerable to buckling under pressure. This likely sets a maximum for the $k$-values of hollow bird bones.

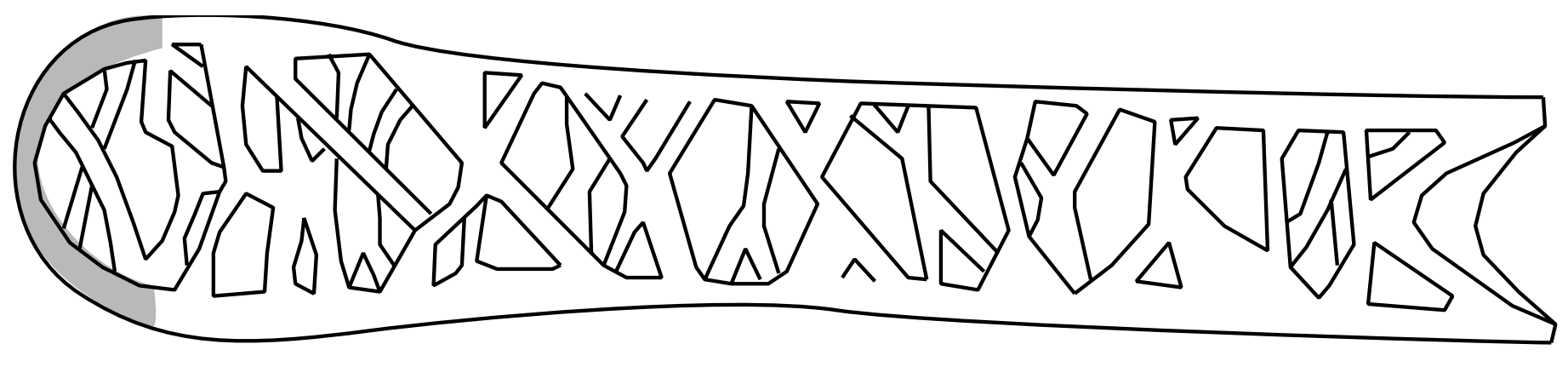

Figure 3. Bird bone

\section{REFERENCES}

McNeill, Alexander R. 1983. Animal Mechanics. Oxford Press. McNeill, Alexander R. 1990. Animals. Cambridge University Press. McNeill, Alexander R. 1996. Optima for Animals. Princeton Press. 